\title{
Brainstem Portion of the Pyramidal Tracts
}

National Cancer Institute

\section{Source}

National Cancer Institute. Brainstem Portion of the Pyramidal Tracts. NCI Thesaurus.

Code C142308.

The segments of the corticospinal and corticobulbar tracts that either traverse or terminate in the brainstem. 